\title{
Idiopathic granulomatous gastritis diagnosed with endoscopic ultrasound-guided fine-needle aspiration: report of a case
}

Authors

Institution
Koh Imbe, Atsushi Irisawa, Goro Shibukawa, Yoko Abe, Akiko Saito, Koki Hoshi, Akane Yamabe, Ryo Igarashi

Department of Gastroenterology, Fukushima Medical University, Aizu Medical Center, Aizuwakamatsu, Japan submitted 26. May 2014 accepted after revision 8. September 2014

\section{Bibliography}

Dol http://dx.doi.org/ 10.1055/s-0034-1390744

Published online: 24.10 .2014

Endoscopy International Open 2014; 02: E259-E261

(c) Georg Thieme Verlag KG Stuttgart . New York E-ISSN 2196-9736

\section{Corresponding author}

Atsushi Irisawa, MD, PhD

Department of

Gastroenterology

Fukushima Medical University

Aizu Medical Center

21-2, Maeda, Tanisawa,

Kawahigashi

Aizuwakamatsu 969-3492

Japan

Fax: +81-242-75-2568

irisawa@fmu.ac.jp
A 71-year-old man in whom a gastric submucosal lesion was found incidentally was referred to our hospital for detailed examination. Esophagastroduodenoscopy showed a submucosal lesion in the body of the stomach. Endoscopic ultrasound revealed a $15-\mathrm{mm}$ hypoechoic round mass with calcifications arising from the muscular layer. Confusing the diagnosis, it resembled a gastroin-

\section{Introduction}

\section{$\nabla$}

Granulomatous gastritis is an extremely rare condition. A granuloma is an organized aggregation of histiocytes, lymphocytes, and plasma infiltrate. When such an organized collection of cells is identified in the stomach, it is designated as granulomatous gastritis, a subtype of chronic gastritis classified according to its cause as infectious, noninfectious, or idiopathic granulomatous gastritis [1-3]. Most cases of granulomatous gastritis in economically developed countries are noninfectious, with the most common causes in adults and children being Crohn disease and sarcoidosis $[2,3]$. In economically developing countries, infectious diseases, particularly infection with Mycobacterium tuberculosis, are the most common cause of granulomatous gastritis [4]. In up to $25 \%$ of cases, no cause of granulomatous gastritis is identifiable [2]. These patients are considered to have idiopathic granulomatous gastritis.

Of the many kinds of gastric submucosal lesions that exist, the most common neoplasm is gastrointestinal mesenchymal tumor (GIMT), especially gastrointestinal stromal tumor (GIST). Because all GISTs have malignant potential, a definitive diagnosis for additional treatment is important. Recently, endoscopic ultrasound (EUS) has progressed to the degree that a diagnosis of GIST can be inferred from the EUS image. However, immunologic staining for c-KIT or CD34 remains necessary for the definitive diagnosis of GIST. Therefore, EUS-guided fine-needle aspiration biopsy (EUS- testinal mesenchymal tumor. Subsequently, endoscopic ultrasound-guided fine-needle aspiration was conducted for definitive diagnosis. Pathologic analysis showed a granuloma. Because this patient had no prior exposure to tuberculosis or Helicobacter pylori infection and had no abnormal laboratory data, this submucosal lesion was diagnosed as idiopathic granulomatous gastritis.

FNA) is necessary [5]. This report describes a case of idiopathic granulomatous gastritis in the form of a submucosal lesion mimicking GIMT on an EUS image. This report is the first describing a case of idiopathic granulomatous gastritis in the form of a submucosal lesion diagnosed by EUS-FNA.

\section{Case report \\ $\nabla$}

A 71-year-old man in whom a gastric submucosal lesion was found incidentally was referred to our hospital for detailed examination. He had no symptoms or significant physical findings. Esophagastroduodenoscopy (EGD) showed a submucosal lesion in the body of the stomach ( $\bullet$ Fig. 1 ). EUS with a $20-\mathrm{MHz}$ miniprobe showed a $15-\mathrm{mm}$ hypoechoic, round mass with calcifications arising from the fourth (muscular) layer ( $\bullet$ Fig. 2 ). Its appearance was confusingly similar to that of a GIMT.

Subsequently, EUS-FNA was performed with a curved linear array scope (GF-UCT260; Olympus Medical Systems, Tokyo, Japan) and a 25-gauge needle (Expect; Boston Scientific, Tokyo, Japan) for a definitive diagnosis ( $\bullet$ Fig.3). Although needling of the small mass was difficult, samples sufficient for histologic analysis were collected in three passes. Hematoxylin and eosin staining showed a granuloma; CD68 immunostaining showed CD68 + cells ( $\bullet$ Fig.4). Consequently, we diagnosed granulomatous gastritis in the form of a submucosal lesion. 


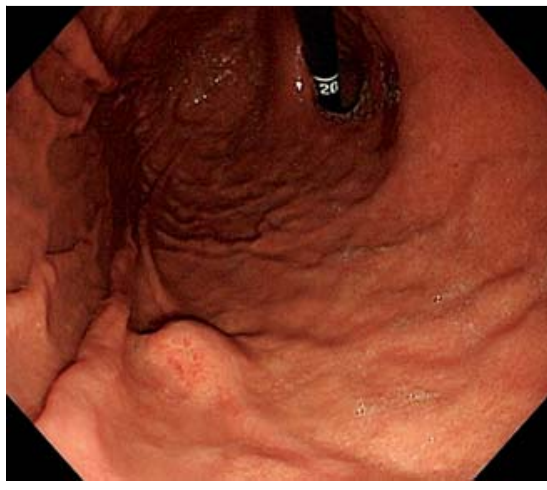

Fig. 1 Image obtained during esophagogastroduodenoscopy showing a submucosal lesion in the body of the stomach.

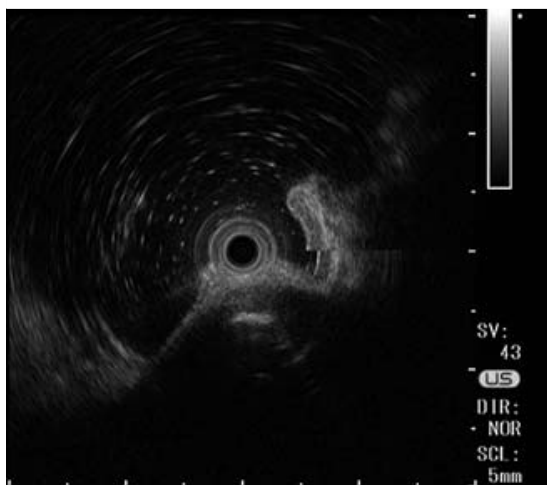

Fig.2 Endoscopic ultrasound image obtained with a $12-\mathrm{MHz}$ miniprobe showing a 15-mm hypoechoic round mass with calcification emerging from the gastric muscular layer.

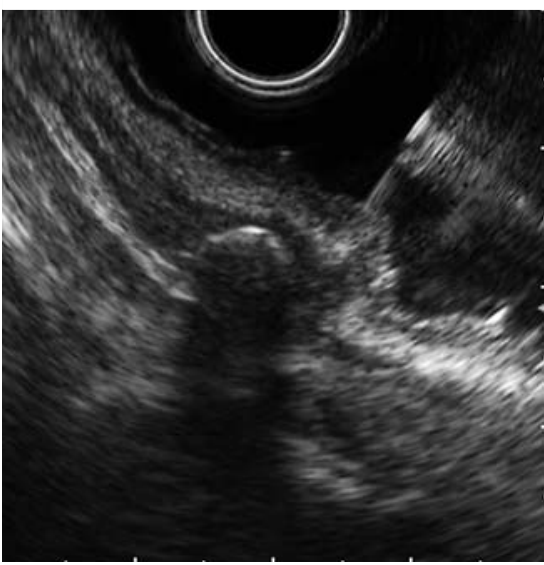

Fig.3 Image of the submucosal lesion obtained during endoscopic ultrasound guided fine-needle aspiration with a 25-gauge needle.

This patient had no symptoms or remarkable history except hypertension and hyperlipidemia. Laboratory investigations revealed normal liver and renal function. The results of a complete blood cell analysis were normal. In addition, results of the QuantiFERON TB-2G test for tuberculosis (Cellestis, Carnegie, Victoria, Australia) were negative. There was no finding of sarcoidosis, and the result of testing for Helicobacter pylori antibody was negative. Because of these clinical findings, we diagnosed this submucosal lesion as idiopathic granulomatous gastritis. The laboratory investigations revealed no symptom or abnormality; therefore, we inferred that we did not have to intervene aggressively in this case. After 6 months, EUS showed no specific changes of idiopathic granulomatous gastritis.
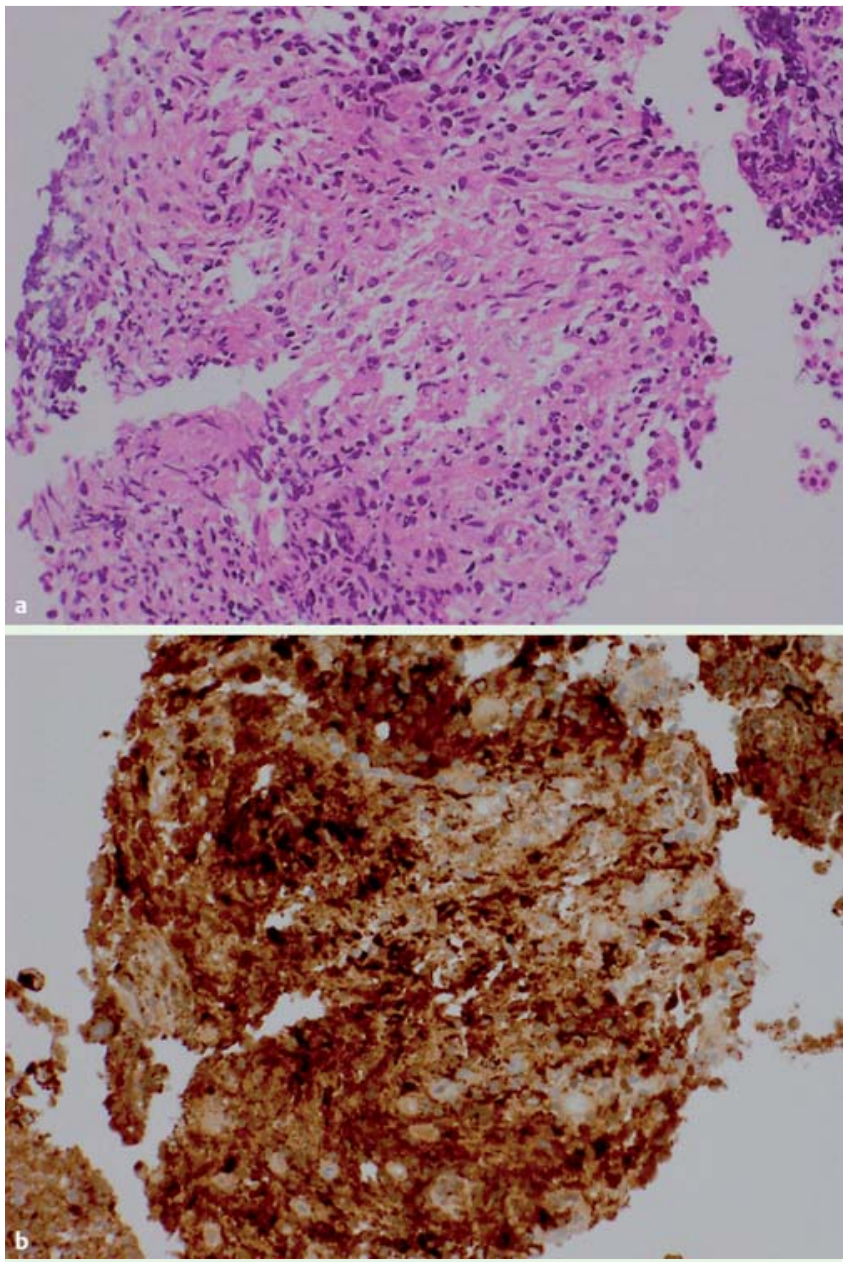

Fig. 4 Histopathologic analysis reveals a granulomatous mass. a Hematoxylin and eosin stain showing a granuloma. b CD68 immunostaining showing a CD68 + cell.

\section{Discussion}

\section{$\nabla$}

Gastric submucosal lesions are usually found incidentally during routine EGD. The differential diagnosis of gastric submucosal lesions includes a number of benign and malignant nonepithelial gastric tumors, intramural vessels, and extrinsic compression by extramural structures [5]. Generally, lipoma, leiomyoma, neurilemmoma, and aberrant pancreas are listed among benign tumors. Although not all GISTs are malignant, GIST is the most important neoplasm with malignant potential in gastrointestinal submucosal lesions [6].

Recently, EUS technology has progressed rapidly. It has become a popular modality for the characterization of digestive disease. Gastrointestinal submucosal lesions are appropriate target lesions for detailed EUS examination because EUS can reveal their localization, size, and consistency better than EGD can. As one example, lipoma is readily diagnosed by its EUS characteristics without the need for histopathology. However, Hwang et al. demonstrated that the presumptive EUS diagnosis agreed with the histologic diagnosis in only $48 \%$ of gastric submucosal lesions [5]. On EUS, mesenchymal tumors, including GISTs, are typically visualized as hypoechoic and homogeneous with a well-defined margin within the fourth layer (muscularis propria) [7]. In addition, small lesions without EUS features of irregular margins, cystic areas, or echogenic foci can be regarded as benign. In the case 
we present here, EUS showed a hypoechoic, round mass with calcification arising from the gastric fourth layer. Calcification is rare in submucosal lesions and is most often visualized in GIST and leiomyoma. Therefore, the EUS findings in our patient resembled those of GIMT. We infer that EUS-FNA was an extremely important modality for making a definitive diagnosis in this patient.

We used a 25-gauge EUS-FNA needle in this case because the target lesion was small. Several reports have indicated that although 22 - and 25-gauge needles are equally effective in terms of diagnostic yield and overall accuracy, the flexibility of a 25-gauge needle is superior to that of a 22-gauge needle $[8,9]$. Because EUS-FNA of a mobile, small gastrointestinal stromal/mesenchymal tumor is typically difficult, special manipulation with full angulation and twisting of the scope is occasionally required. Therefore, a 25-gauge needle should be used, particularly for a small GI-SMT.

In this case, a submucosal lesion was eventually diagnosed as granulomatous gastritis with EUS-FNA. Granulomatous gastritis is a subtype of chronic gastritis that is classified according to its cause as infectious, noninfectious, or idiopathic [1-3]. Most cases of granulomatous gastritis occur in patients with Crohn disease, sarcoidosis, or infection. Because this patient had no symptom or abnormality, we diagnosed idiopathic granulomatous gastritis. Granulomatous gastritis presenting as a submucosal lesion is generally a rare condition, which underscores the necessity of using EUS-FNA in assessing gastrointestinal submucosal lesions. Unfortunately, in our retrospective review of the EUS findings, we were unable to identify specific EUS features of granulomatous gastritis in the form of a submucosal lesion.

As described above, idiopathic granulomatous gastritis is generally not included in the differential diagnosis of gastric submucosal lesions. It is difficult to diagnose granulomatous gastritis based on the EUS findings alone. Actually, a surgical case of idio- pathic granulomatous gastritis resembling a GIST has been reported [10]. Idiopathic granulomatous gastritis has no malignant potential. Therefore, surgical resection for treatment is unnecessary. When a gastric submucosal lesion is found, granulomatous gastritis should be included in the differential diagnosis. EUSFNA is recommended for additional management.

\section{Competing interests: None}

\section{References}

1 Shapiro JL, Goldblum JR, Petras RE. A clinicopathologic study of 42 patients with granulomatous gastritis. Is there really an "idiopathic" granulomatous gastritis? Am J Surg Pathol 1996; 20: 462 - 470

2 Ectors NL, Dixon MF, Geboes KJ et al. Granulomatous gastritis: a morphological and diagnostic approach. Histopathology 1993; 23: 55-61

3 Maeng L, Lee A, Choi K et al. Granulomatous gastritis: a clinicopathologic analysis of 18 biopsy cases. Am J Surg Pathol 2004; 28: 941 - 945

4 Kamani L. Granulomatous gastritis: a diagnostic dilemma? Singapore Med J 2008; 49: e222 - e224

5 Hwang JH, Saunders MD, Rulyak SJ et al. A prospective study comparing endoscopy and EUS in the evaluation of GI subepithelial masses. Gastrointest Endosc 2005; 62: 202-208

6 Sepe PS, Brugge WR. A guide for the diagnosis and management of gastrointestinal stromal cell tumors. Nat Rev Gastroenterol Hepatol 2009; 6: $363-371$

7 Landi B, Palazzo L. The role of endosonography in submucosal tumours. Best Pract Res Clin Gastroenterol 2009; 23: 679-701

8 Affolter KE, Schmidt RL, Matynia AP et al. Needle size has only a limited effect on outcomes in EUS-guided fine needle aspiration: a systematic review and meta-analysis. Dig Dis Sci 2013; 58: 1026-1034

9 Vilmann P, Săftoiu A, Hollerbach $S$ et al. Multicenter randomized controlled trial comparing the performance of 22 gauge versus 25 gauge EUS-FNA needles in solid masses. Scand J Gastroenterol 2013; 48: $877-883$

10 Niitsu H, Tanabe K, Tokumoto $\mathrm{N}$ et al. Idiopathic granulomatous gastritis resembling a gastrointestinal stromal tumor. Case Rep Gastroenterol 2012; 6: $502-509$ 